\title{
A Triptolide Loaded HER2-Targeted Nano-Drug Delivery System Significantly Suppressed the Proliferation of HER2-Positive and BRAF Mutant Colon Cancer
}

Ayimukedisi Yalikong ${ }^{1,2, *}$ Xu-Quan $\mathrm{Li}^{1,3, *}$

Ping-Hong Zhou ${ }^{1,2}$

Zhi-Peng $\mathrm{Qi}^{1,2}$

Bing $\mathrm{Li}^{1,2}$

Shi-Lun Cai ${ }^{1,2}$

Yun-Shi Zhong ${ }^{1,2}$

'Endoscopy Center, Zhongshan Hospital of Fudan University, Shanghai, 200032, People's Republic of China; ${ }^{2}$ Endoscopy Research Institute of Fudan University, Shanghai, 200032, People's Republic of China;

${ }^{3}$ Shanghai Henlius Biopharmaceuticals Co., Ltd., Shanghai, 200033, People's Republic of China

*These authors contributed equally to this work

Correspondence: Shi-Lun Cai; Yun-Shi Zhong

Zhongshan Hospital of Fudan University, I80 Fenglin Road, Shanghai, 200032,

People's Republic of China

Email caishilun1988@qq.com;

zhongyunshi@yahoo.com
This article was published in the following Dove Press journal:

International Journal of Nanomedicine

Background: Colon cancer (CRC) was a malignant tumor and there were about $25 \%$ of patients with tumor metastasis at diagnosis stage. Chemotherapeutic agents for metastatic CRC patients were with great side effects and the clinical treatment results of advanced CRC were still not satisfactory. Human epidermal growth factor receptor 2 (HER2) is overexpressed in some CRC patients and is an effective target for CRC patient treatment. Anti-HER2 therapy had a beneficial role in the treatment of HER2-positive metastatic CRC with fewer side effects. CRC patients with BRAF mutations were resistant to HER2 antibodies treatment. Therefore, there was an urgent need to develop new therapeutic agents.

Methods: HER2 targeted nanoparticles (TPLNP) drug delivery system loading triptolide (TPL) were prepared and identified. The effects of TPLNP and free TPL on cell viability, targeting and cell cycle progression on HT29 (BRAF mutation) with HER2 overexpression, were evaluated by Cell Counting Kit-8 (CCK8), Fluorescence Activating Cell Sorter (FACS) and immunofluorescence methods, respectively. The anti-tumor efficacies of TPLNP were evaluated in subcutaneous xenograft model of colon cancer and the survival rate, tumor volume, liver and kidney indexes of tumorbearing mice were measured.

Results: TPLNP was small in nanosize $(73.4 \pm 5.2 \mathrm{~nm})$ with narrow size distribution $(\mathrm{PDI}=0.15 \pm 0.02)$ and favorable zeta potential $(\mathrm{pH}=9.6$, zeta potential: $-57.3 \pm 6.69 \mathrm{mV}$; $\mathrm{pH}=7.0$, zeta potential: $-28.7 \pm 5.1 \mathrm{mV} ; \mathrm{pH}=5.6$, zeta potential: $-21.1 \pm 4.73 \mathrm{mV}$ ). Comparing with free TPL treatment group, TPLNP developed stranger colon cancer-killing efficiency in a dose- and time-dependent manner detected with CCK8 method; achieved good in vitro colon cancer targeting detected with flow cytometry and immunofluorescence experiments; enhanced more HT29-HER2 apoptosis and induced more cell cycle arrested in G1-S phase detected with FACS in vitro. As for in vivo antitumor response, TPLNP remarkably inhibited the growth of colon cancer in the colon cancer xenograft model, significantly improved the survival rate and did not exhibit significant liver and kidney toxicity in contrast with free TPL in vivo.

Conclusion: TPLNP was effectively against colon cancer with HER2 overexpression and BRAF mutation in pre-clinical models. In summary, the TPLNP appeared to be a promising treatment option for CRC in clinical application based on improved efficacy and the favorable safety profile.

Keywords: colon cancer, HER2, triptolide, BRAF mutation 


\section{Introduction}

Colon cancer (CRC) is a malignant tumor derived from the epithelial cells of the colonic mucosa and is one of the common malignant tumors in gastrointestinal tract. ${ }^{1}$ CRC is with high incidence, and once CRC patients enter the advanced stage, the survival period of within five years will reduce as low as $14 \%$ and the life quality of CRC is relatively poor. About $25 \%$ of CRC patients suffer tumor metastasis at diagnosis stage and about $50-60 \%$ of CRC patients transferred into metastatic stage. ${ }^{2}$ Although some patients with advanced CRC can undergo radical surgery, most of the metastatic patients suffer from incurable diseases $^{3}$ and the treatment for this population is focused on palliative systemic treatment. Although great progress has been achieved in the early diagnosis, treatment and management of CRC, the clinical treatment results of advanced CRC are still not satisfactory. Chemical anticancer drugs such as Oxaliplatin and 5-FU have been recognized to decrease recurrence and prolong survival time, ${ }^{4}$ especially for advanced patients. ${ }^{5}$ But the development of systemic toxicity and drug resistance caused by chemical drugs are the main obstacles to the successful treatment of CRC. ${ }^{6}$ Most commonly, irinotecan is used in combination with fluorouracil infusion (5-FU) and leucovorin (LV) as the first-line therapy for metastatic CRC. Irinotecan can be used as chemotherapy for CRC patients who cannot tolerate 5 -FU. ${ }^{7}$ The efficacy of irinotecan is prevented by high drug cytotoxicity, serious impact on the bone marrow (such as neutropenia) and the gastrointestinal tract (such as diarrhea). Despite the highly effective chemotherapeutics for the treatment of CRC, their low specificity usually produces a series of dose-limited side effects, such as nausea, hair loss or vomiting. ${ }^{6}$ Therefore, irinotecan-based chemotherapy is generally reserved for wellbeing patients who can resist adverse effects. These adverse effects limited its usage in patients with underperformance. Coupled with high treatment costs, such interventions may greatly reduce the quality of life of CRC patients.

As a member of the human epidermal growth factor receptor (EGFR) family, human epidermal growth factor receptor 2 (HER2) is overexpressed and related to the increased risk of recurrence and poor prognosis of metastatic gastric cancer. ${ }^{8}$ Several studies have evaluated the relationship between the HER2 expression profile and the development of CRC patients. ${ }^{9}$ HER2 antibody was the first-line therapy for the resistance to epidermal growth factor receptor to rescue the patients. ${ }^{10,11}$ Until now, the reports and clinical data have evidenced that anti-HER2 therapy has potential advantages in the treatment of HER2-positive metastatic CRC without BRAF or RAS mutation. ${ }^{12,13}$ Although combination therapy including anti-HER2 antibody (trastuzumab) and chemotherapy can improve the remission rate, the duration of response to trastuzumab lasts only 5-9 months. ${ }^{9,14,15}$ However, due to the existence of resistant mechanisms, patients become difficult to benefit from anti-EGFR therapy. The mechanisms of HER2 therapy resistance mainly lie in the mutations in BRAF, KRAS, NRAS and PI3KCA. ${ }^{16,17}$ It was proved that the patients with BRAF mutations, especially V600E mutations did not react with anti-HER2 treatment. ${ }^{8,18,19}$ Therefore, the development of novel antiHER2 therapeutic agents for CRC treatment is an urgent need.

Triptolide (TPL) as an inhibitor of topoisomerase I has been used in the treatment of CRC. It has been proved that TPL demonstrated good suppression effects on multiple tumor cell lines with high potency (under $\mathrm{nM}$ level) in vitro and could suppress the tumor growth in vivo. ${ }^{20-22}$ Before reaching the clinical potential, many obstacles must be conquered, such as low solubility, narrow treatment window and systematic toxicity. ${ }^{23}$ In special cases, longterm use of TPL can cause serious adverse reactions, ${ }^{22}$ such as hepatic injury with increased serum alanine aminotransferase (ALT), aspartate aminotransferase (AST), and kidney damage with ascendant serum creatinine (CRE) and urea nitrogen (URE) and the incensement in oxide dismutase (SOD).

The development of nanoparticle-based cancer treatment strategies has made substantial progress in pharmacology, reducing the side effects of cytotoxic drugs, improving their efficacy, solubility, pharmacokinetics and bio-distributions. ${ }^{24}$ During the past five decades, series nanoparticles of different shapes, sizes and chemical properties have shown high efficiency in encapsulating different types of anti-cancer drugs. ${ }^{25,26}$ These first-generation anti-cancer nanoparticles used the enhanced penetration and retention (EPR) effects provided by tumor blood vessels and lymphatic drainage to passively reach tumor tissues. ${ }^{27}$ This allows nanoparticles to spread and accumulate in cancer cells and improve the therapeutic effects. ${ }^{13,28}$ Nano-drug delivery system has emerged and been a very valuable treatment strategy for the treatment 
of CRC cancer. The targeted drug delivery systems take advantage of protein biomarkers specifically overexpressed on the tumor surface and pack considerable amounts of anti-tumor drugs to achieve targeting release of anti-tumor drugs and reduced systemic side effects. ${ }^{29-33}$ In this study, we prepared a HER2-targeted nano-drug delivery system for the treatment of colon cancer with HER2 positive and BRAF mutation and we test the antitumor effects of the drug delivery system in vitro and in vivo.

\section{Materials}

Triptolide (TPL) was bought from Shanghai Tongtian Biotech company (Shanghai, China). Polyglutamic acid (PGA, mean molecular weight, 20,000 Daltons) was from Nanjing Zijigang biotech company (Nanjing, China). AntiHER2 antibody was from Bioxcell (New Hampshire, USA). Fluorescein isothiocyanate (FITC) labeled kit was from Invitrogen (Carlsbad, CA). 4'6-Diamidino-2-phenylindole (DAPI), Methanol, Dimethyl sulfoxide (DMSO), 1-Ethyl3-(3-dimethylaminopropyl) carbodiimide hydrochloride (EDCI) and L-phenylalanine ethyl ester (Phe) were purchased from Aladdin Company (Shanghai, China). HER2 overexpressed Lentivirus was from Hanheng Company (Shanghai, China). Cell Counting Kit-8 (CCK8) was from Dojindo (Tokyo, Japan). Streptomycin and penicillin (P/S), cell culture medium (DMEM) and fetal calf serum (FBS) were purchased from Hyclone (Logan, UT, USA). Apoptosis detection kit (AnnexinV-APC and propidium iodide) was purchased from BD (Franklin Lake, NJ, USA).

\section{Cells and Animals}

HT29 cell line was purchased from the Chinese Academy of Sciences (Shanghai, China). Cells were cultured in DMEM (Gibco, USA) with $10 \%(\mathrm{v} / \mathrm{v})$ fetal bovine serum (Gibco, USA) and $1 \% \mathrm{P} / \mathrm{S}$ in $37^{\circ} \mathrm{C}$ with $5 \% \mathrm{CO} 2$. BALB/c nude mice were obtained from Shanghai Laboratory Animal Company (Shanghai, China).

\section{Methods}

\section{HER2 Overexpression Cell Line Preparation}

HT29 cells were BRAF mutation cell line with HER2 relative low expression and HT29 cells infected with HER2 overexpressed Lentivirus. HER2 positive cells were selected with puromycin $(5 \mu \mathrm{g} / \mathrm{mL})$ for 3 weeks. HT29 and HT29-HER2 were incubated with anti-HER2 antibody and detected with FACS and analysis with BD Flowjo X (Franklin Lake, NJ, USA).

\section{Synthesis of HER2 Targeted Nanomedicine}

HER2 antibody was labeled with FITC using a commercial kit (Invitrogen, Carlsbad, CA) in line with the manufacturer's protocol. PGA-L-Phe nanoparticles were prepared in line with the reported method. ${ }^{34,35}$ The procedures were conducted as the following: HER2 antibody (1mg) and polyglutamic acid (77mg) and L-Phe $(20 \mathrm{mg})$ in the mixture water consisting of EDCI $(50 \mathrm{mg})$ was agitated overnight on a magnetic stirrer at $4^{\circ} \mathrm{C}$ for $8 \mathrm{~h}$. And the products were obtained by centrifugation (12000rpm, 5min) and freeze-drying. The products (25mg) were dissolved in DMSO and mixed with TPL (10mg) or not to form mixture solution. And then, the solution was added into $10 \mathrm{~mL}$ of the aqueous solution dropwise in a $100 \mathrm{~mL}$ flask with stirring at $300 \mathrm{rpm}$. Organic solvents were removed by centrifugation (12000rpm, 10min) to collect the produced empty nanoparticle (ENP) or TPL loaded nanoparticle (TPLNP). And then, the final products were washed with distilled water for three times to remove the surfactant.

\section{Character of HER2 Targeted Nano-Medicine}

The mean particle size, size distribution and polydispersity index (PDI) of the nanoparticles were detected with Malvern laser particle size analyzer (Malvern, UK) at $25^{\circ} \mathrm{C}$. The samples measured by dynamic light scattering (DLS) were prepared by diluting the nanoparticle suspension with appropriately distilled deionized water and filtered with a syringe filter.

\section{TPL Loading Efficiency Detection}

High-performance liquid chromatography (HPLC, Agilent 1100) was used to check the amount of TPL incorporated into the nanoparticles with a reversed-phase Lichrospher ODS C18 column. $10 \mathrm{mg}$ of TPL-loaded nanoparticles were dissolved into $1 \mathrm{~mL}$ of DMSO to release TPL and the solution was dried with a rotary evaporator. And then, TPL was extracted with methanol. To detect the TPL concentration, the solution obtained after filtration and was put into a vial for HPLC detection. The mobile phase consisting a mixture of methanol-water $(52 / 48, \mathrm{v} /$ v) maintained with a flow rate of $1.0 \mathrm{~mL} / \mathrm{min}$. A variable 
wavelength detector was used to detect the column effluent at $218 \mathrm{~nm}$, and the column temperature was kept at $30^{\circ} \mathrm{C}$. The drug entrapment rate (ER) is calculated by the following formula: the $\mathrm{ER}=\mathrm{M} 1 / \mathrm{M} 2$. M1 means the measured TPL quality detected with HPLC, M2 indicated the total TPL quality performed for the entrapment detection. The TPL entrapment efficiency (EE) was counted by the following formula: the $\mathrm{EE}=\mathrm{C} 1 / \mathrm{C} 2$. $\mathrm{C} 1$ represented the measured TPL content packaged in TPLNP, C2 represented the total quality of TPLNP.

\section{Cell Viability Assay}

The effect of TPLNP on cell viability was assessed by CCK8. HT29 overexpressed HER2 were seeded in a 96 well plate overnight, and then the supernatant was removed and treated with TPLNP (containing 100, 50, 25, 12.5, 6.25nM TPL) or TPL (100, 50, 25, 12.5, 6.25nM TPL) for 48h. CCK8 reagent $(10 \mu \mathrm{L})$ was appended into each well and incubated for another $2 \mathrm{~h}$ at $37^{\circ} \mathrm{C}$. The optical density (OD) at $570 \mathrm{~nm}$ of each well was measured by BioTek PEOCH2 system (Vermont, USA).

\section{Cell Cycle Analysis}

To explore the affluence of TPLNP and TPL on HT29HER2 cell cycle, HT29-HER2 overexpressed cells treated with PBS, ENP, TPLNP (containing 200nM TPL) or TPL $(200 \mathrm{nM})$ for $5 \mathrm{~h}$ and collected with centrifugation $(800 \mathrm{~g}$, $5 \mathrm{~min}$ ), and then culture for another $48 \mathrm{~h}$. Later, cells were obtained with centrifugal collection and fixed with $70 \%$ ice-cold ethanol for $4 \mathrm{~h}$, and stained with propidium iodide $(50 \mu \mathrm{M})$ for $30 \mathrm{~min}$ at room temperature, then examined with a BD Fortessa flow cytometer (Franklin Lake, NJ). DNA histograms were produced with BD Flowjo X (Franklin Lake, NJ, USA).

\section{In vitro Targeting Test}

In vitro targeting test was detected by flow cytometry and immunofluorescence methods. HT29-HER2 overexpressed cells were added to a 24-well plate and mixed with PBS, TPL (200mM), ENP (equal to TPLNP) or TPLNP (containing $200 \mathrm{nM} \mathrm{TPL}$ ), and incubated at $37^{\circ} \mathrm{C}$ for $30 \mathrm{~min}$. The cells were collected at $600 \mathrm{~g}$ centrifugal force, washed twice, and then re-suspended in $400 \mu \mathrm{L}$ FACS buffer and analyzed on the Fortessa FACS flow cytometer (BD, NJ). TPLNP target ability on HT29-HER2 cells was detected with immunofluorescence method. HT29 cells were fused to $80 \%$ to $90 \%$ in 24 wells plates with PBS, TPL (200nM), ENP (equal to TPLNP) or TPLNP (containing 200nM
TPL) and incubated in $37^{\circ} \mathrm{C}$ for $1 \mathrm{~h}$, and fixed with $4 \%$ paraformaldehyde. The nucleus is stained with DAPI solution. The laser power and photomultiplier gain were kept constant and images of stained cells were acquired.

\section{The Apoptosis Detection}

HT29-HER2 cells $\left(1 \times 10^{6}\right)$ were seeded in a 6-well plate and treated with $200 \mathrm{nM}$ free TPL or TPLNP (containing $200 \mathrm{nM}$ TPL) for $4 \mathrm{~h}$, and then washed with PBS to remove free TPL or TPLNP and incubated for another $24 \mathrm{~h}$. Then, double staining was performed using Annexin V-APC/PI kit (Franklin Lake, NJ, USA) to quantify the rate of apoptosis. PI $(1 \mu \mathrm{L})$ and Annexin V-APC $(1 \mu \mathrm{L})$ were added into cell mixtures at $4^{\circ} \mathrm{C}$ for 30 minutes. Then, cells were mixed with $400 \mu \mathrm{L}$ flow cytometer buffer. The apoptosis rates of diverse groups were checked with BD Fortessa FACS and analyzed by BD FlowJo X software (Franklin Lake, NJ, USA).

\section{In vivo Antitumor Effects}

The in vivo antitumor effects of TPLNP were performed in HT29-HER2 bearing adult female BALB/c nude mice ( $n=6 /$ group), all the mice were raised in a specific pathogen-free (SPF) environment and all the animal experiments were approved and conducted in accordance with the Animal Experiment Ethics Committee of Zhongshan Hospital, Fudan University. Mice were supplied with clean, comfortable living conditions, adequate feed and clean drinking water. Anesthesia was performed before subcutaneous tumor or intravenous injection. As the experiment was over, the experimental animals were sacrificed and euthanasia must be implemented according to humanitarian principles. HT29-HER2 cells $\left(1 \times 10^{6} /\right.$ mice $)$ were harvested and subcutaneous injection was performed in nude mice on upper back. As the tumor volume approached to near $100 \mathrm{~mm}^{3}$, the tumor-bearing mice were randomly assigned into 4 groups according to body weights. PBS, TPL $(0.9 \mathrm{mg} / \mathrm{kg}$ ), TPLNP (containing $0.9 \mathrm{mg} / \mathrm{kg} \mathrm{TP}$ ), and ENP were injected into each group through tail venous injection three times per week for 4 weeks, respectively. The tumor volume changes of the tumor-bearing mice were recorded and all the mice were euthanized as the experiment was over and the antitumor efficiency of the TPLNP was measured by weighing the final tumor. The tumor volumes were counted with the following formula: $E=a \times b^{2} / 2(a=$ length of tumor, $b=$ width of tumor). 


\section{In vivo Hepatorenal Toxicity Evaluation}

At the end of the experiment, blood samples were taken from the orbital cavity and collected in serum collection tubes and blood serum samples of each mouse were acquired by centrifugal collection $(800 \mathrm{~g}, 10 \mathrm{~min})$. Serum urea nitrogen (BUN) and creatinine (CRE) levels and the activities of alanine aminotransferase (ALT) and aspartate aminotransferase (AST) of the mice were detected with corresponding diagnostic kits (Nanjing Jiancheng, China) in line with the manufacturer's instruction.

\section{Statistical Analysis}

For the data between the two groups is determined by the Student's $t$-test. A two-way analysis of variance (ANOVA) determined the statistical significance of more than 3 groups, a $P<0.05$ is considered with statistics difference.

\section{Results}

\section{HER2 Overexpression Cell Line Detection}

The HER2 expression level on HT29 and HT29-HER2 cells was detected with FACS method. As shown in Figure 1A, HT29 was a HER2 relatively low expression cell line. HT29 transformed into HER2 positive cell line, after the infection of HER2 lent-virus. HT29-HER2 cell line was used for the later experiments.

\section{The Character of TPLNP}

Detected with DLS, the mean particle size of TPLNP was $73.4 \pm 5.2 \mathrm{~nm}$ (Figure 1B); The PDI is $0.15 \pm 0.2$, and the zeta potential of TPLNP altered with the changes of $\mathrm{pH}$ values $(\mathrm{pH}=9.6$, zeta potential: $-57.3 \pm 6.69 \mathrm{mV} ; \mathrm{pH}=7.0$, zeta potential: $-28.7 \pm 5.1 \mathrm{mV} ; \mathrm{pH}=5.6$, zeta potential: $-21.1 \pm 4.73 \mathrm{mV}$ ). The nanoparticles below $100 \mathrm{~nm}$ are beneficial for the entry of tumor tissue due to the EPR effects. In the water phase, the zeta potential boundary of stable particles is generally considered to be higher than $+30 \mathrm{mV}$ or lower than $-30 \mathrm{mV}$. The zeta potential of TPLNP was suitable to form a stable dispersion state and avoided the risk of drug precipitation. TPL entrapment rate (ER) and entrapment efficiency (EE) in TPLNP measured by HPLC were $36.8 \%$ and $11.4 \%$, respectively. As shown in Supplementary Figure 1, under the neutral conditions. TPLNP showed a good sustained-release manner both in PBS and normal saline. The median maximum release time $\left(\mathrm{t}_{1 / 2}\right)$ of TPLNP was about $24 \mathrm{~h}$ in PBS and $30 \mathrm{~h}$ in saline. In vitro sustained release experiment indicated that TPLNP had a good sustained release manner. The sustained-release dosage form can reduce the frequency of
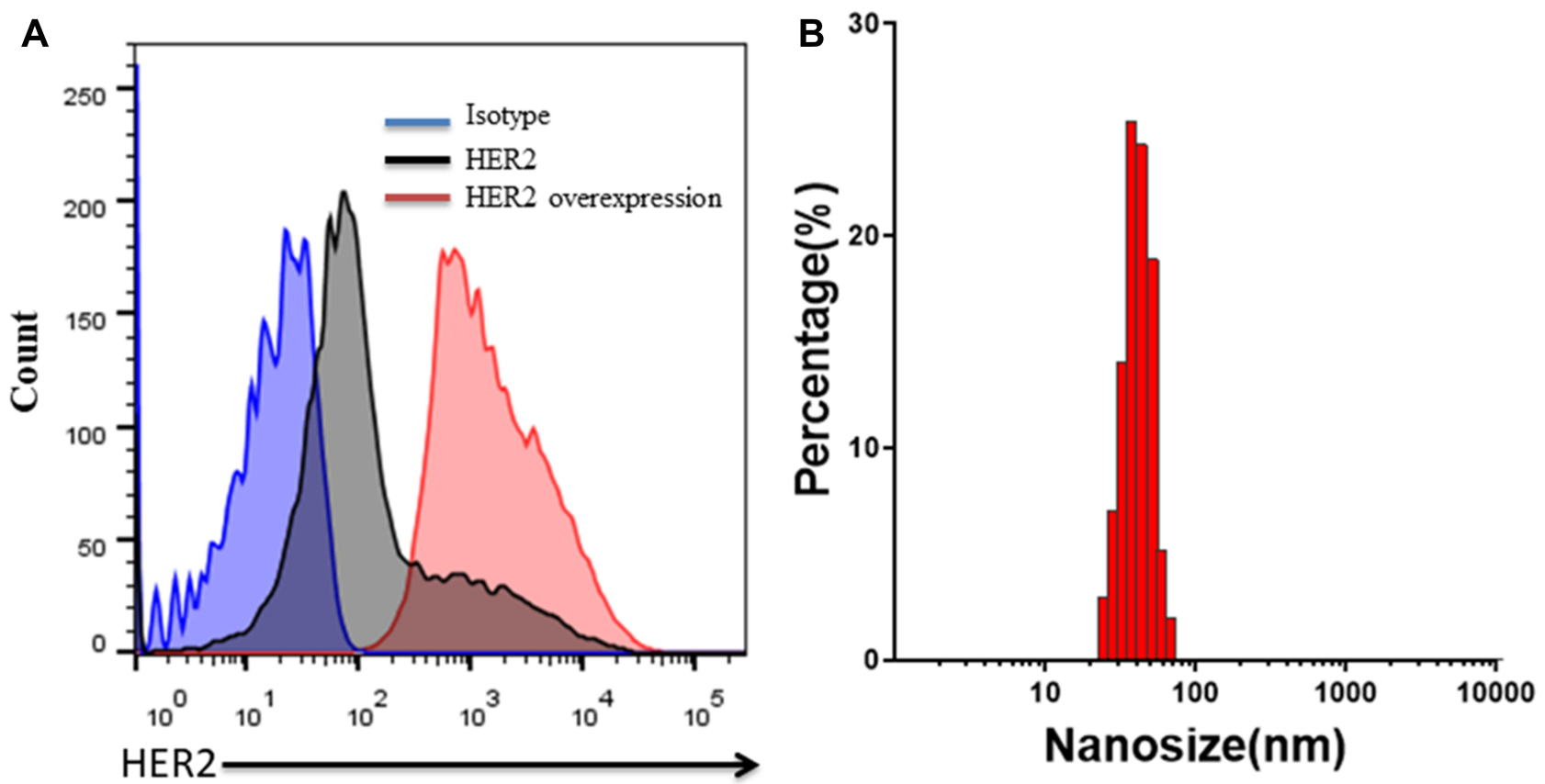

Figure I (A) HER2 expression levels of HT29 and HT29-HER2 cells detected with FACS method, (B) The nano-size of TPLNP detected with DLS method. 

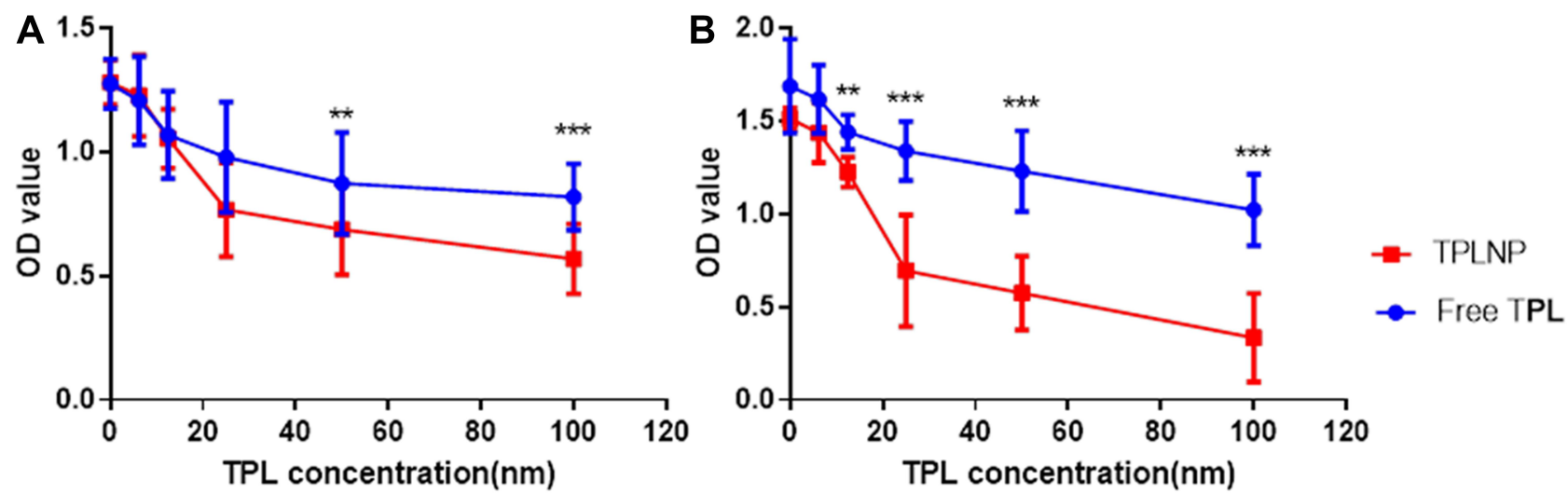

Figure 2 The cytotoxicity of TPLNP on HT29-HER2 colon cancer cells detected with CCK8 method. HT29-HER2 cells incubated with TPLNP or TPL for 4h and washed with PBS to remove the free TPL and TPLNP and cultured for another $24 \mathrm{~h}(\mathbf{A})$ and $48 \mathrm{~h}(\mathbf{B})$, respectively. $* * P<0.01, * * * P<0.001$.

drug administration, maintain good blood concentration, and improve the compliance of patients.

\section{TPLNP Enhanced Cytotoxicity on HER2 Positive and Arrested the Cell Cycle}

CCK8 was an effective reagent for cell proliferation detection. Data showed that TPL decreased the viability of HT29-HER2 colon cancer cells with a dose relationshipdependent manner (Figure 2A and B). After a short period of incubation, the nano-drug delivery system could adhere to the surface of the cells with high expression of HER2, and enter into the cells through receptor-mediated endocytosis. TPL could be released in the cells and achieve an effective tumor cell killing effect. Only two doses (50nM and $100 \mathrm{nM}$ ) showed statistical difference in the first $24 \mathrm{~h}$, and the statistical difference varied from $10 \mathrm{nM}$ to $100 \mathrm{nM}$ on 48h. Comparing with the free TPL treatment group, the half inhibitory concentration (IC50) of TPL group (Table 1) was $386.24 \pm 59.3 \mathrm{~nm}$ (24h) and $168.3 \pm 23.4 \mathrm{~nm}$ (48h) which was much higher than TPLNP treatment group. We evaluated the cell cycle arrest effects of TPLNPs on colon cancer. We used propidium iodide (PI) staining to analyze cell cycle and to identify the cycle arrest effects of TPLNP on HT29-HER2. After the

Table I The Half Inhibition Concentration (IC50) of TPL and TPLNP on HT29-HER2 Cells

\begin{tabular}{|l|l|c|}
\hline \multirow{2}{*}{ Incubation Period(h) } & \multicolumn{2}{|c|}{ IC50 Value(nM) } \\
\cline { 2 - 3 } & Free TPL & TPLNP \\
\hline $24 \mathrm{~h}$ & $386.24 \pm 59.3$ & $128 \pm 79.5$ \\
\hline $48 \mathrm{~h}$ & $168.3 \pm 23.4$ & $38.9 \pm 10.3$ \\
\hline
\end{tabular}

treatment of PBS or ENP, cells were with more G2 phase Figure 3A ( $\mathrm{G} 1=57.14, \mathrm{~S}=24.26, \mathrm{G} 2=17.09 \%)$ and Figure 3B ( $\mathrm{G} 1=55.78 \%, \mathrm{~S}=25.83, \mathrm{G} 2=16.29 \%)$. Went through the treatment of TPL (Figure 3C), cells were with less G2 phase $(\mathrm{G} 1=57.74 \%, \mathrm{~S}=37.72, \mathrm{G} 2=4.54 \%)$. Data showed that TPLNP treatment induced cell accumulation at G0/ G1 cycle $(\mathrm{G} 1=84.73 \%, \mathrm{~S}=24.23, \mathrm{G} 2=0 \%)$ in Figure 3D. TPLNP also induced HT29-HER2 cancer apoptosis. PBS, ENP, free TPL and TPLNP cultured with HT29-HER2 cancer in a short period to allow TPLNP to adhere to the cell surface. Apoptosis rate was identified by Annexin-V and PI staining. Double positive indicated late apoptosis; PI single staining represented necrotic cells; Annexin-V single staining meant early apoptotic cells and live cells were Annexin-V and PI double negative staining. The cell apoptotic rates counted the proportions of the upper right (Annexin-V and PI double positive) and lower right quadrants (Annexin-V positive). After the treatment of PBS (Figure 4A) and ENP (Figure 4B), the apoptosis rate was considerably low $(10.2 \%$ in PBS treatment group, $11.2 \%$ in ENP treatment group). Comparing with PBS and ENP treatment group, free TPL treatment (Figure 4C) significantly induced cell apoptosis $(25.74 \%)$. TPLNP treatment showed the most lethal effect $(52.9 \%)$ on HT29-HER2 positive cell line (Figure 4D).

\section{TPLNP Achieved HER2 Targeting}

\section{Function}

HER 2 targeting was detected by FACS and immunofluorescence. FITC was labeled on the drug carrier system. PBS, ENP, TPLNP and free TPL were incubated with HT29-HER2 cells, separately. After the treatment of PBS (Figure 5A) or free TPL (Figure 5B), fluorescence 

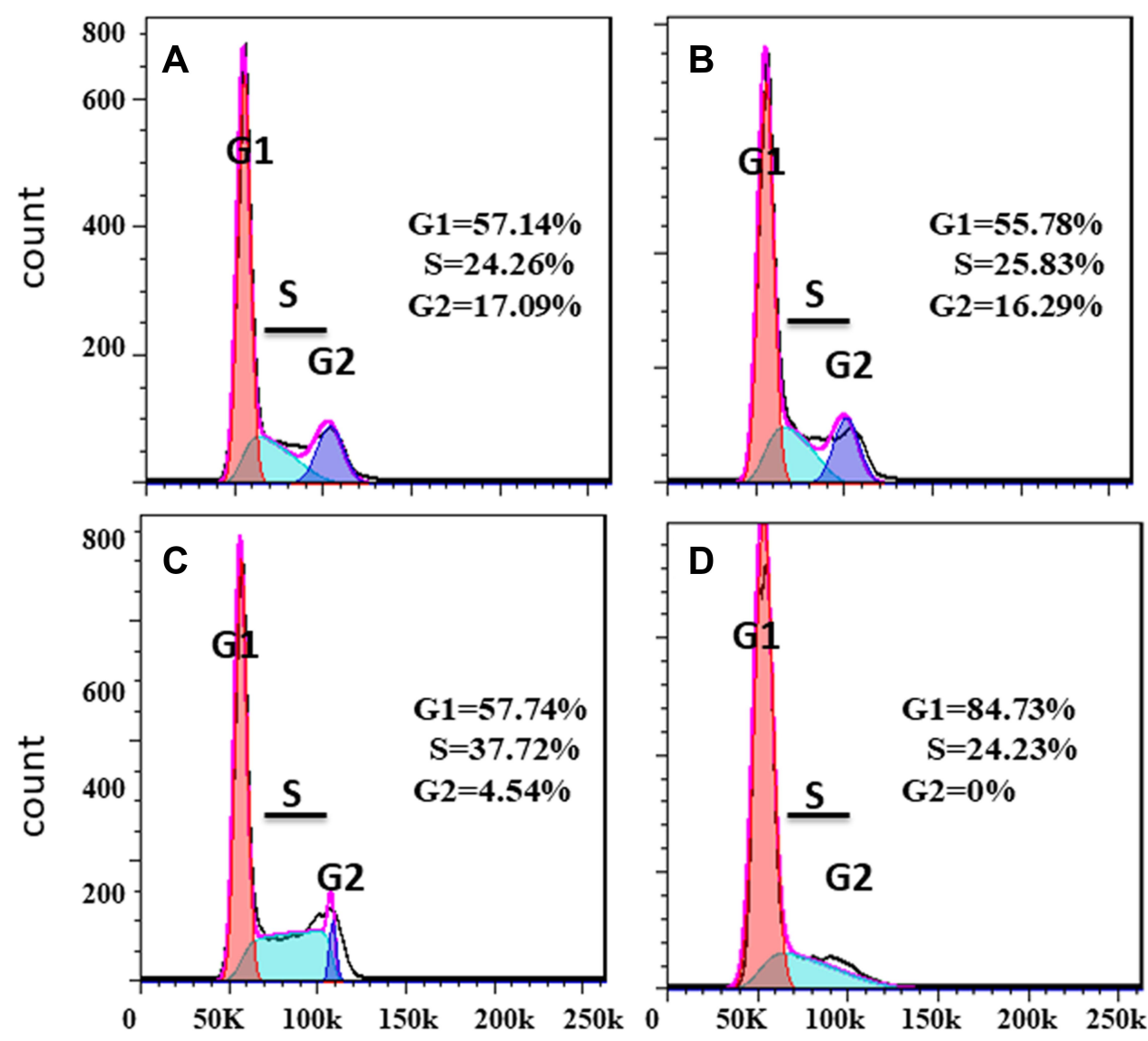

Figure 3 The cell cycle was detected with FACS method after TPLNP treatment. HT29-HER2 cells incubated with PBS, ENP, TPL and TPLNP for 4h and then washed with PBS and cultured for another 24h. Cells of different groups were collected and incubated with PI to detect cell cycle. (A) PBS treatment group, (B) ENP treatment group, (C) TPL treatment group, (D) TPLNP treatment group.

intensity was relatively low detected with FACS (the positive rates of HER2 are $3.45 \%$ in PBS treatment group and $3.5 \%$ in TPL treatment group). After the treatment of ENP (Figure 5C) and TPLNP (Figure 5D), the curves shifted to the right (the positive rates of HER2 are 99.5\% in ENP treatment group and $98.5 \%$ in TPLNP treatment group). Higher fluorescence intensity indicated that the TPLNP and ENP achieved HER2 targeting. HER2 targeting was further detected with immunofluorescence method; no FITC staining was detected in PBS (Figure 6A) or TPL (Figure 6B) treatment groups. However, under the staining of ENP (Figure 6C) and TPLNP (Figure 6D), cells were stained with FITC fluorescence.

\section{TPLNP Improve Mice Survival Rate}

The survival rate of mice is an important indicator of drug safety. After TPL treatment, half amount of mice died because of the systemic toxicity of the TPL and no mice died during the treatment in TPLNP group and one mouse died in PBS group (Figure 7A). Comparing with free TPL or PBS treatment group, the use of TPLNP significantly improved the animal survival rate.

\section{TPLNP Suppressed Cancer Development}

In order to evaluate the therapeutic effect of TPLNP on the colon cancer, we applied the mouse CRC model. Eight-week-old female nude mice were involved in each group. After tumor formation, TPLNP (containing $0.9 \mathrm{mg} / \mathrm{kg} \mathrm{TP})$, TPL (0.9 mg/kg), empty drug carrier (ENP) or PBS were administered intraperitoneally three times per week. The mice were euthanized and the sizes of colon tumors were analyzed. The tumor volume kept increasing with time elapsed. After the treatment of TPL and TPLNP, the slower tumor growth rate was detected (Figure 7C). Larger tumor volumes were detected in the ENP or PBS groups. The data showed that TPLNP treatment significantly reduced the total tumor burden (Figure 7D). 


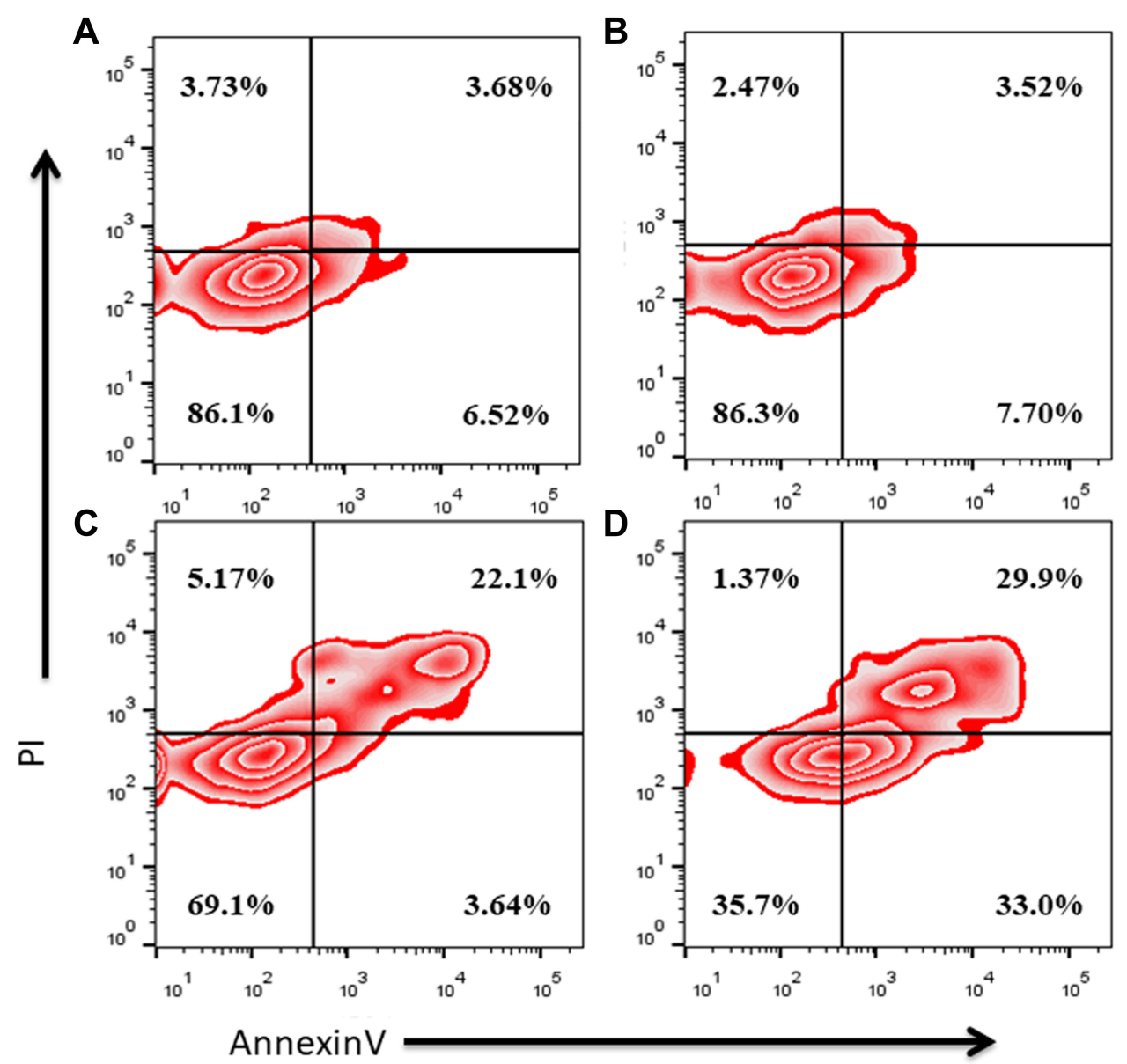

Figure 4 The apoptosis rates of HT29-HER2 cells were detected with FACS method after TPLNP treatment. HT29-HER2 cells incubated with PBS, ENP, TPL and TPLNP for $4 \mathrm{~h}$ and then washed with PBS and cultured for another 24h. Cells of different groups were collected and incubated with PI and Annexin-V to detect the apoptosis rates. (A) PBS treatment group, (B) ENP treatment group, (C) TPL treatment group, (D) TPLNP treatment group.

\section{TPLNP Decreased Hepatorenal Toxicity} Induced with TPL

TPL was with systematic toxicity and we determined its toxicity by detecting indicators such as liver, kidney toxicity. After administration of the nanoparticles, serums from 4 groups were analyzed. It was observed that compared with the PBS group, free TP caused nephrotoxicity and liver toxicity with increased levels of ALT, AST, CRE and BUN. High levels of serum AST and ALT were indicators of liver injury, which were caused by hepatocyte necrosis. The ALT and AST levels of tumor-bearing mice treated with TPL increased significantly (Figure 7B), which was consistent with the liver and kidney toxicity of TPL. However, treatment with TPLNP did not increase liver toxicity in tumorbearing mice (Figure 7B). Similarly, the level of BUN, CRE in serum as an index of nephrotoxicity proved that TPLNP nanoparticle treatment could significantly induce renal toxicity comparing with free TPL treatment.

\section{Discussion}

Although considerable progress has been made on $\mathrm{CRC}$, for patients with metastatic colon cancer, surgery; radiotherapy and chemotherapy are mostly palliative treatments and there is still no effective treatment. HER2 is a common metastatic colon cancer marker, and its high expression generally indicates the low patient's survival rate with recognized carcinogenic effects. ${ }^{36}$ Although the HER2 positive rate is relatively low among the CRC patients, the role of HER2 in malignant tumor development and progression is defined as the high dependence of tumor cell proliferation and tumor metastasis. HER2 consists of extracellular domain, trans-membrane domain and intracellular domain containing the tyrosine kinase (RTK) activity. HER2 was with active RTK activity and the overexpression of HER2 also makes HER2 molecules constitutively activate. Anti-HER2 antibodies inhibit HER2 phosphorylation to some extent and decrease the expression of HER2 on the 


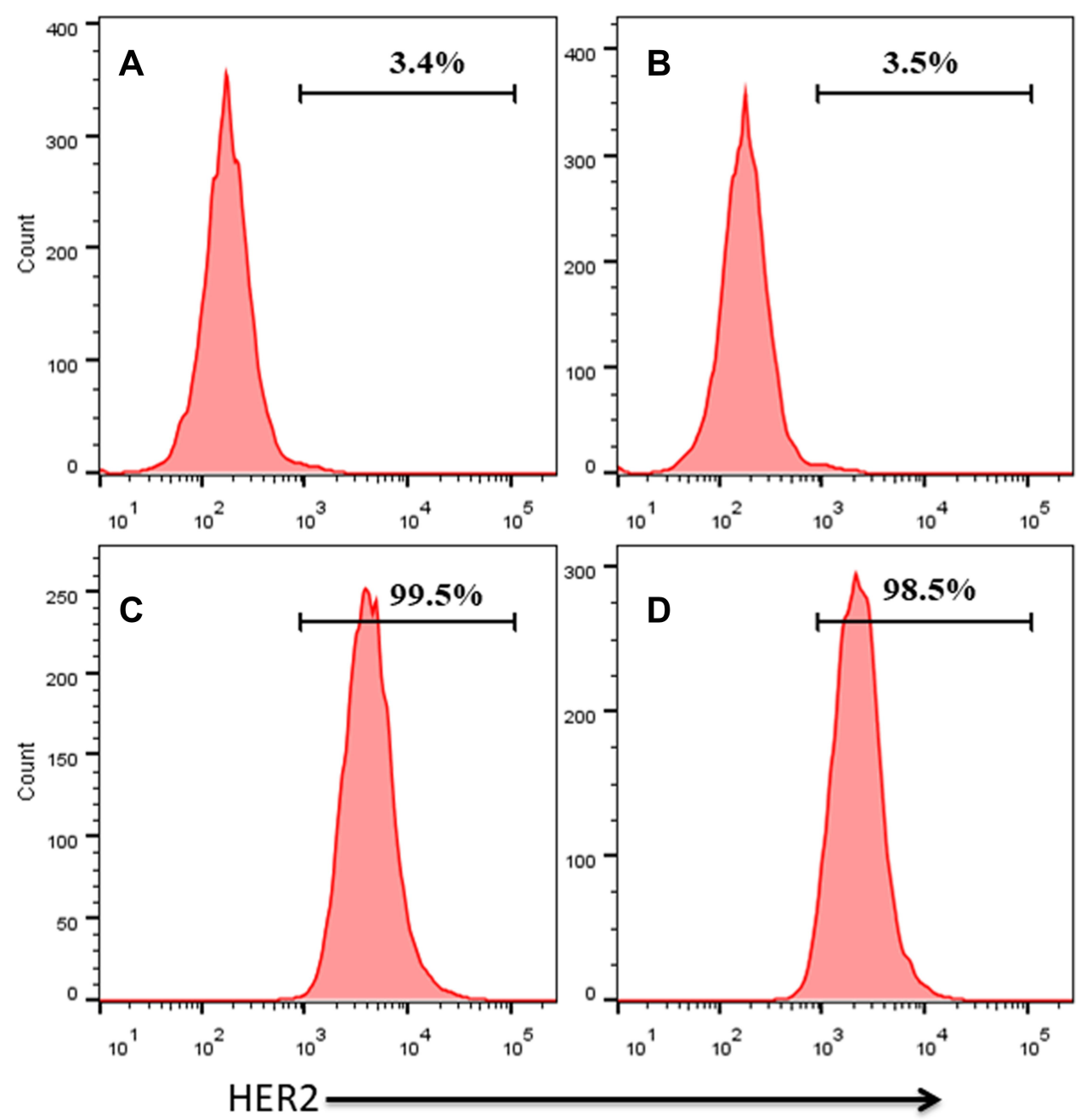

Figure 5 FACS method was used to test cell targeting ability of TPLNP. HT29-HER2 cells incubated with PBS (A), TPL (B), ENP (C) and TPLNP (D) for 30min and washed with PBS. The fluorescence intensities of each group were detected with FACS method.

cell surface. Nano-targeted drug system can carry abundant antitumor drugs to kill tumor cells which is different from the inhibitory effect of antibodies on HER2 overexpressed tumor cells. As a drug carrier contacts with the cell surface, a large number of tumors killing or inhibitory drugs will be transported into the tumor through receptor-mediated endocytosis. TPLNP produced its antitumor activity like the HER2 targeted antibody conjugated drug (ADC) with a great affinity for HER2 and kill cells by exerting cytotoxic effect which could overcome resistance due to decreased HER2 expression. ${ }^{37}$

The usage of liposomes as a drug delivery system containing HER2 realized the targeting of HER2 overexpressed tumor cell and the inhibition of target cell proliferation. ${ }^{38}$ However, the instability of liposome formulations is a problem that has to be considered, which affects the release kinetics of the drug from the nano- system. ${ }^{39}$ HER2 antibody fixed dendrimers realized effective delivery of drugs HER2 overexpressed tumor cells. ${ }^{40}$ However, the time-consuming development process and nano-scale steric hindrance make it difficult to generate higher generation dendrimers. ${ }^{41}$ TPLNP drug delivery is a kind of micelle which owns superiorities over other drug delivery systems. TPLNP possesses excellent stability in vivo. TPLNP is composed of hydrophobic and hydrophilic parts which formed nano-spheres by self-assembly and enclosed water-insoluble or hydrophobic drugs in the core. The hydrophobic core can dissolve water-insoluble molecules, while the hydrophilic shell can protect the internal regions of the drug molecules in the biological system from adverse environmental conditions such as the influence of $\mathrm{pH}$ value, the non-specific adsorption of drugs and the degradation of hydrolytic enzymes. ${ }^{42}$ Besides, the nano-size of micelle is easy to control; the hydrophilic 
DAPI
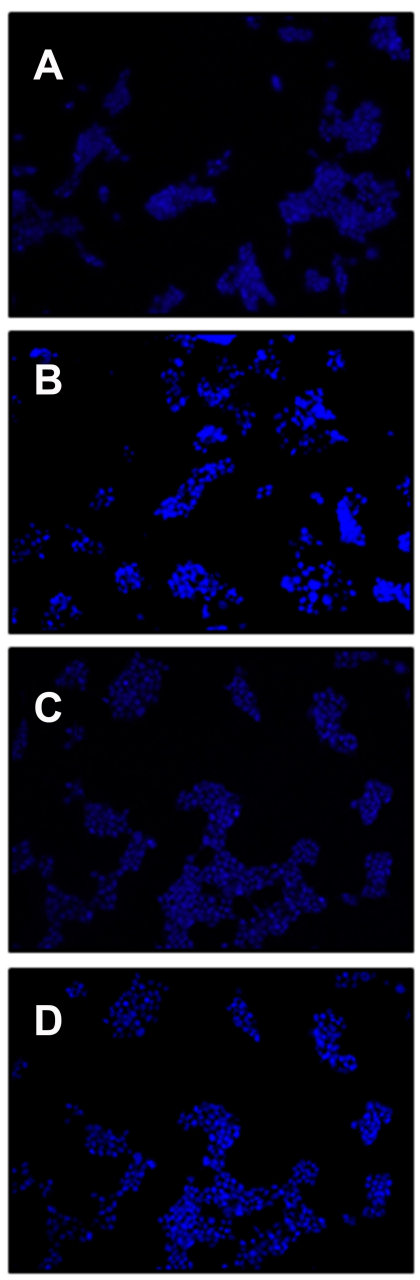

FITC
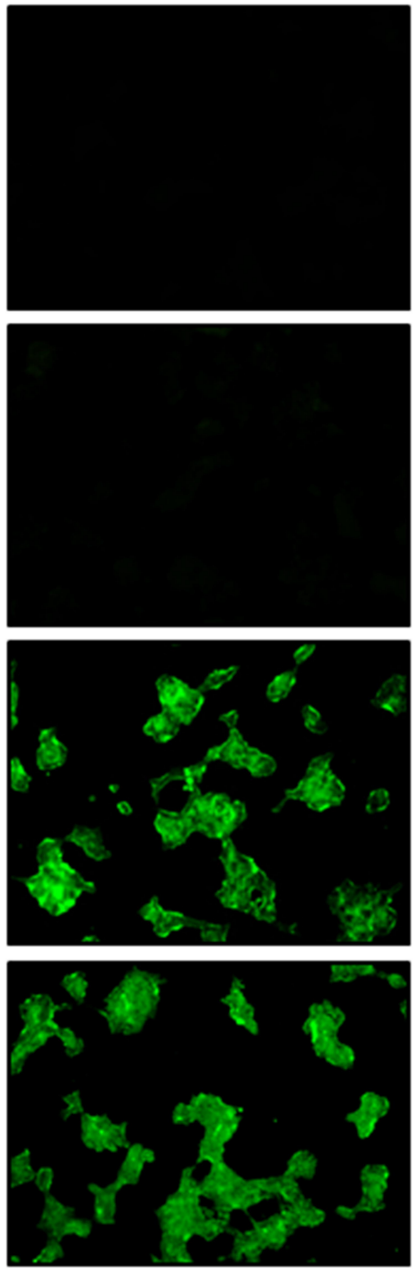

Merge
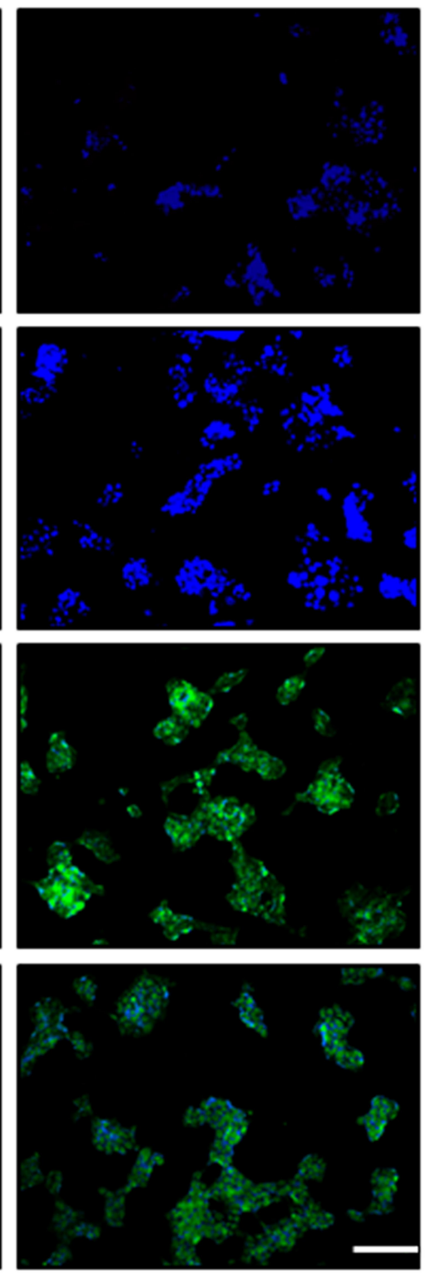

Figure 6 The cell-targeting ability of TPLNP detected with immunofluorescence method. HT29-HER2 cells cultured in 24-well plate and incubated with PBS (A), TPL (B), ENP (C) and TPLNP (D) for 30min; cells were washed with PBS and then the nuclei were stained with DAPI. Lastly, the images of the cells were recorded under fluorescence microscopy.

shell was easy to modify with multiple functional groups and the self-assembly manner made the synthesis more convenient.

Anti-HER2 antibodies could bind to the extracellular domain of the HER2 to prevent the activation and suppress the activation of downstream signaling. Anti-HER2 antibodies have improved clinical treatment effects for metastatic CRC patients. ${ }^{43}$ So far, the Food and Drug Administration has approved two anti-EGFR drugs cetuximab and panitumumab for the treatment of colorectal cancer, so EGFR can be used as an important and clinically relevant therapeutic target for colon cancer. However, the duration of response to anti-HER2 antibodies lasts short. Patients with BRAF, KRAS, NRAS and PI3KCA mutations are resistant to anti-EGFR therapy. In the current study, colon cancer cell line HT29 cell was reported with BRAF mutation and was infected with HER2 overexpressed lentivirus to simulate clinical colon cancer status. Targeted nanoparticles can take advantage of differential expression molecules on the surface of tumor cells and effectively release cytotoxic drugs. In this research, we developed a HER2 targeted nanodrug delivery system. HER2 targeted TPL delivery system made full use of the HER2 overexpression on the surface CRC cells and were able to bind to it tightly. The in vitro experiment demonstrated that TPLNP could realize the HER2 target detected with FACS and immunofluorescence methods. TPLNP could also realize targeting cytotoxicity detected with CCK8 method and apoptosis detected with FACS. What's more, TPLNP could also realize the arresting of cell cycles on HT29HER2. The use of nano-delivery system for targeted 

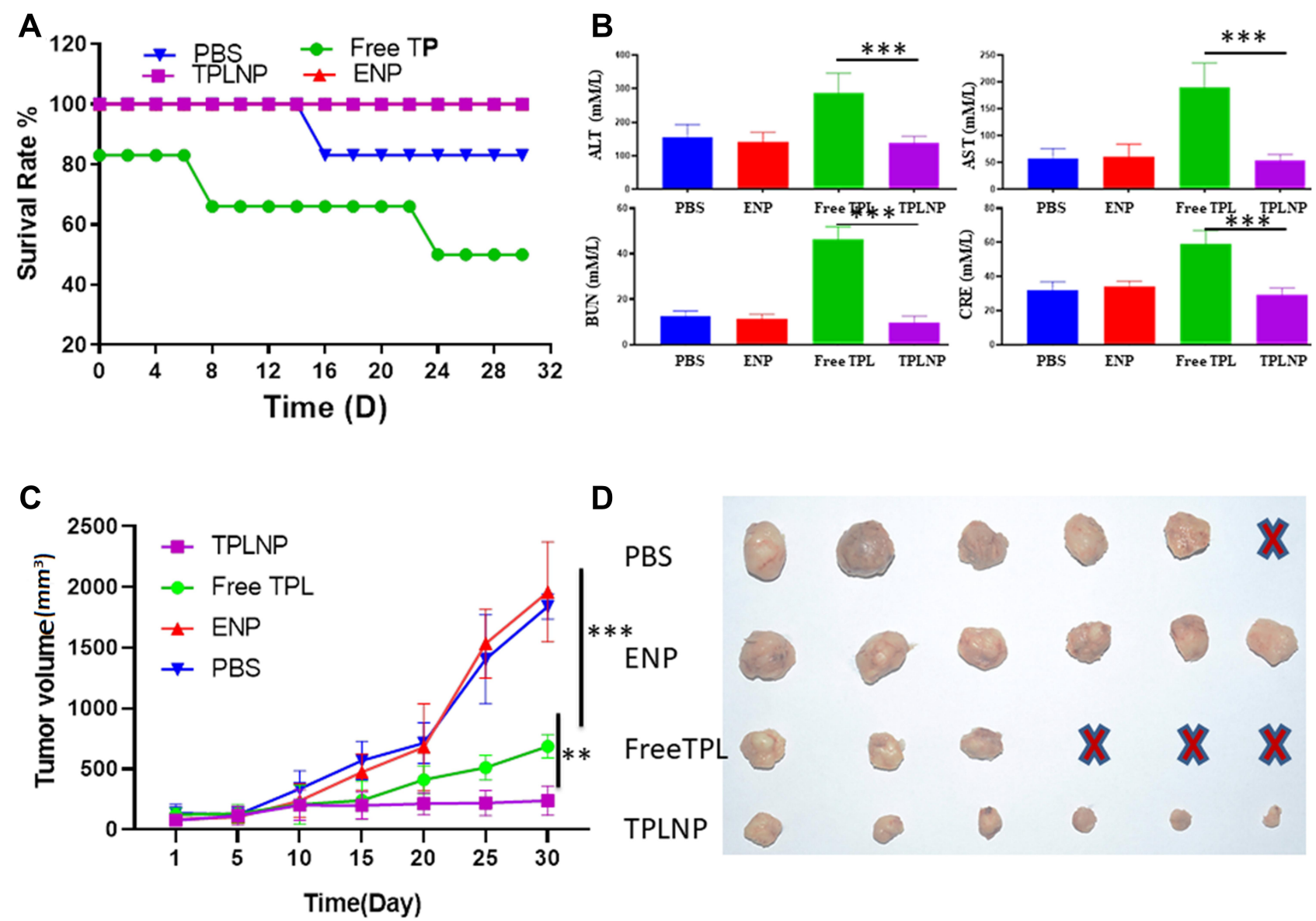

D
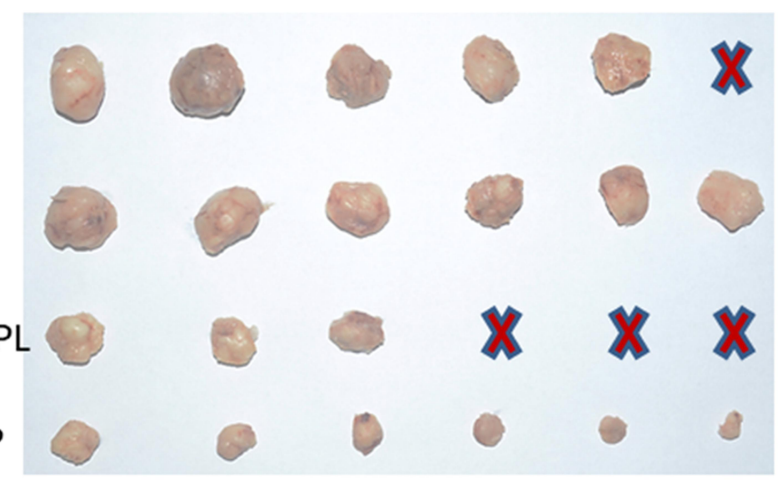

Figure 7 The antitumor effects of TPLNP in vivo. (A) survival rate detection, (B) liver and kidney function detection, (C) tumor volume changes of the tumor-bearing mice, (D) the tumor images acquired from different groups. In vivo antitumor effects of TPLNP were performed in HT29-HER2 bearing adult female BALB/c nude mice ( $\mathrm{n}=6$ / group). HT29-HER2 cells ( $1 \times 10^{6} /$ mice) were harvested and subcutaneous injection was performed in nude mice on the upper back. The tumor-bearing mice were randomly assigned into 4 groups according to their body weights. Tumor-bearing animals received treatments of PBS, free TPL (0.9 mg/kg), TPLNP (containing $0.9 \mathrm{mg} / \mathrm{kg}$ TP), or ENP, through tail venous injection every 2 days per week and the treatment last for 4 weeks. Mice were sacrificed after the last treatment. $* * P<0.0 \mathrm{I}$, $* * * P<0.00 \mathrm{I}$.

killing not only reduced the side effects of drugs to the body, greatly improved the targeted killing effect on tumor tissues, which was a strong support for the effectiveness of HER2 antibodies. The major function of HER2 antibody was to target HER2 positive tumor cells and the cytotoxic effect was mainly through TPL. TPLNP has the potential to treat colon cancer cells with HER2 positive expression that are either wild type or with RAS/BRAF mutation.

The poor water solubility of TPL and seriously side effects severely hindered its clinical effectiveness. The water solubility of TPL was significantly improved by nano-drug delivery system, with high drug loading efficiencies. We demonstrated that TPLNP inhibited the proliferation of colon cancer with HER2 overexpression and BRAF mutation in a nude mouse model in vivo. Intravenous injection of the TPLNP on a colon cancer model also demonstrated improved pharmacokinetics this was the enhanced antitumor efficacy, increased survival and the reduced toxicity in vivo compared to the free TPL. Our research strongly suggested that TPLNP is promising treatments for colon cancer with HER2 overexpression and BRAF mutation.

Ado-trastuzumab emtansine (T-DM1) was an antibody-drug conjugate (ADC) which linked trastuzumab to microtubule inhibitor DM1, with a thioether compound that realized active chemotherapy to HER2 positive tumor cells. ${ }^{44}$ Although T-DM1 could realize the treatment of colon cancer, the nano-drug delivery system could carry abundant drugs to achieve targeted drug delivery than ADC, especially TPL could kill tumor cells at the nM level which could demonstrate a stronger tumor suppression effect in the treatment of colon cancer.

As for the in vivo safety, AST and ALT levels were used as indicators of liver abnormalities, and BUN and CRE levels were used as indicators of kidney function. TPLNP 
significantly reduced the liver and kidney toxicity of TPL to mice. At the same time, the survival rate of the mice treated by the nano-drug delivery system was also significantly improved, and there was no death of the mice appeared. Here, we introduced the latest developments in targeted nanoparticles that oppose CRC and discussed the potential use of potential ligands and cellular target treatment strategies for CRC. This study has important clinical application value for CRC patients with high expression of HER2 and BRAF mutation.

Except for colon cancer, the overexpression of HER2 also appears in about $15-30 \%$ of breast cancers and $10-30 \%$ of gastric cancers and other cancers such as lung cancer, ovarian cancer, bladder cancer, and head and neck cancer. ${ }^{37,45}$ What's more, TPL inhibits the growth of cancer cells and exhibits preclinical anti-tumor activity in a variety of gastrointestinal cancers (such as gastric cancer, liver cancer and pancreatic cancer) and other tumors (such as lung cancer, breast cancer, ovarian cancer and prostate cancer). ${ }^{46,47}$ Thus, TPLNP is with the promising to broaden its preclinical and clinical antitumor application in different types of tumors.

\section{Acknowledgments}

This work was supported by grants from the Youth Program of National Natural Science Foundation of China (grant no. 81702305), Zhongshan Hospital of Fudan University (grant no. 2019ZSYXGG02). The funders had no role in the study design, data collection/analysis, decision to publish, or preparation of the manuscript.

\section{Disclosure}

Xu-Quan $\mathrm{Li}$ is an employee of Shanghai Henlius Biopharmaceuticals Co., Ltd. The authors report no other potential conflicts of interest for this work.

\section{References}

1. Cancer Genome Atlas Network. Comprehensive molecular characterization of human colon and rectal cancer. Nature. 2012;487 (7407):330-337. doi:10.1038/nature11252

2. Tricoli JV, Boardman LA, Patidar R, et al. A mutational comparison of adult and adolescent and young adult (AYA) colon cancer. Cancer. 2018;124(5):1070-1082. doi:10.1002/cncr.31136

3. Wagner AD, Syn NL, Moehler M, et al. Chemotherapy for advanced gastric cancer. Cochrane Database Syst Rev. 2017;8(8):Cd004064.

4. Bommer U-A, Vine KL, Puri P, et al. Translationally controlled tumour protein TCTP is induced early in human colorectal tumours and contributes to the resistance of HCT116 colon cancer cells to 5-FU and oxaliplatin. Cell Commun Signal. 2017;15(1):9. doi:10.1186/ s12964-017-0164-3
5. Schmoll HJ, Twelves C, Sun W, et al. Effect of adjuvant capecitabine or fluorouracil, with or without oxaliplatin, on survival outcomes in stage III colon cancer and the effect of oxaliplatin on post-relapse survival: a pooled analysis of individual patient data from four randomised controlled trials. Lancet Oncol. 2014;15 (13):1481-1492. doi:10.1016/S1470-2045(14)70486-3

6. Brungs D, Aghmesheh M, de Souza P, et al. Safety and efficacy of oxaliplatin doublet adjuvant chemotherapy in elderly patients with stage III colon cancer. Clin Colorectal Cancer. 2018;17(3):e549e555. doi:10.1016/j.clcc.2018.05.004

7. Shah MA, Renfro LA, Allegra CJ, et al. Impact of patient factors on recurrence risk and time dependency of oxaliplatin benefit in patients with colon cancer: analysis from modern-era adjuvant studies in the Adjuvant Colon Cancer End Points (ACCENT) database. J Clin Oncol. 2016;34(8):843-853. doi:10.1200/JCO.2015.63.0558

8. Greally M, Kelly CM, Cercek A. HER2: an emerging target in colorectal cancer. Curr Probl Cancer. 2018;42(6):560-571. doi:10.1016/j.currproblcancer.2018.07.001

9. Siena S, Sartore-Bianchi A, Marsoni S, et al. Targeting the human epidermal growth factor receptor 2 (HER2) oncogene in colorectal cancer. Ann Oncol. 2018;29(5):1108-1119. doi:10.1093/annonc/ mdy 100

10. Ingold Heppner B, Behrens H-M, Balschun K, et al. HER2/neu testing in primary colorectal carcinoma. Br J Cancer. 2014;111 (10):1977-1984. doi:10.1038/bjc.2014.483

11. Weiner LM, Dhodapkar MV, Ferrone S. Monoclonal antibodies for cancer immunotherapy. Lancet. 2009;373(9668):1033-1040. doi:10.1016/S0140-6736(09)60251-8

12. Satoh T, Bang Y-J, Gotovkin EA, et al. Quality of life in the trastuzumab for gastric cancer trial. Oncologist. 2014;19(7):712-719. doi:10.1634/theoncologist.2014-0058

13. Piktel E, Niemirowicz K, Wątek M, Wollny T, Deptuła P, Bucki R. Recent insights in nanotechnology-based drugs and formulations designed for effective anti-cancer therapy. J Nanobiotechnology. 2016;14(1):39.

14. Xie YH, Chen YX, Fang JY. Comprehensive review of targeted therapy for colorectal cancer. Signal Transduct Target Ther. 2020;5 (1):22.

15. Meric-Bernstam F, Hurwitz H, Raghav KPS, et al. Pertuzumab plus trastuzumab for HER2-amplified metastatic colorectal cancer (MyPathway): an updated report from a multicentre, open-label, phase 2a, multiple basket study. Lancet Oncol. 2019;20(4):518-530. doi:10.1016/S1470-2045(18)30904-5

16. Li ZN, Zhao L, Yu L-F, et al. BRAF and KRAS mutations in metastatic colorectal cancer: future perspectives for personalized therapy. Gastroenterol Rep (Oxf). 2020;8(3):192-205. doi:10.1093/ gastro/goaa022

17. Al-Shamsi HO, Jones J, Fahmawi Y, et al. Molecular spectrum of KRAS, NRAS, BRAF, PIK3CA, TP53, and APC somatic gene mutations in Arab patients with colorectal cancer: determination of frequency and distribution pattern. J Gastrointest Oncol. 2016;7 (6):882-902. doi:10.21037/jgo.2016.11.02

18. Saridaki Z, Saegart X, De Vriendt V, et al. KRAS, NRAS, BRAF mutation comparison of endoscopic and surgically removed primary CRC paired samples: is endoscopy biopsy material adequate for molecular evaluation? Br J Cancer. 2015;113(6):914-920. doi:10.1038/bjc.2015.307

19. Afrăsânie V-A, Marinca MV, Alexa-Stratulat T, et al. KRAS, NRAS, BRAF, HER2 and microsatellite instability in metastatic colorectal cancer - practical implications for the clinician. Radiol Oncol. 2019;53(3):265-274. doi:10.2478/raon-2019-0033

20. Clawson KA, Borja-Cacho D, Antonoff MB, et al. Triptolide and TRAIL combination enhances apoptosis in cholangiocarcinoma. J Surg Res. 2010;163(2):244-249. doi:10.1016/j.jss.2010.03.067

21. Yang S, Chen J, Guo Z, et al. Triptolide inhibits the growth and metastasis of solid tumors. Mol Cancer Ther. 2003;2(1):65-72. 
22. Wang JM, Chen R-X, Zhang -L-L, et al. In vivo protective effects of chlorogenic acid against triptolide-induced hepatotoxicity and its mechanism. Pharm Biol. 2018;56(1):626-631. doi:10.1080/ 13880209.2018.1527370

23. Zhao J, Xie $\mathrm{C}$, $\mathrm{Mu} \mathrm{X}$, et al. Metabolic alterations in triptolide-induced acute hepatotoxicity. Biomed Chromatogr. 2018;32(10):e4299. doi:10.1002/bmc.4299

24. Luo YY, Shi J-F, Chen L, et al. [Research progress on triptolide functionalized nanoparticulate drug delivery systems for tumor treatment]. Zhongguo Zhong Yao Za Zhi. 2019;44(21):4566-4572. doi:10.19540/j.cnki.cjcmm.20190804.308Article in Chinese.

25. Bahrami B, Hojjat-Farsangi M, Mohammadi H, et al. Nanoparticles and targeted drug delivery in cancer therapy. Immunol Lett. 2017;190:64-83.

26. Nabil G, Bhise K, Sau S, et al. Nano-engineered delivery systems for cancer imaging and therapy: recent advances, future direction and patent evaluation. Drug Discov Today. 2019;24(2):462-491. doi:10.1016/j.drudis.2018.08.009

27. Dawidczyk CM, Kim C, Park JH, et al. State-of-the-art in design rules for drug delivery platforms: lessons learned from FDA-approved nanomedicines. J Control Release. 2014;187:133-144. doi:10.1016/j. jconrel.2014.05.036

28. Bertrand N, Wu J, Xu X, Kamaly N, Farokhzad OC. Cancer nanotechnology: the impact of passive and active targeting in the era of modern cancer biology. Adv Drug Deliv Rev. 2014;66:2-25.

29. Pangburn HA, Ahnen DJ, Rice PL. Sulindac metabolites induce proteosomal and lysosomal degradation of the epidermal growth factor receptor. Cancer Prev Res (Phila). 2010;3(4):560-572. doi:10.1158/1940-6207.CAPR-09-0159

30. D’Huyvetter M, De Vos J, Xavier C, et al. 131 I-labeled anti-HER2 camelid $\mathrm{sdAb}$ as a theranostic tool in cancer treatment. Clin Cancer Res. 2017;23(21):6616-6628. doi:10.1158/1078-0432.CCR-17-0310

31. Naruphontjirakul P, Viravaidya-Pasuwat K. Development of anti-HER2-targeted doxorubicin-core-shell chitosan nanoparticles for the treatment of human breast cancer. Int $J$ Nanomedicine. 2019;14:4105-4121. doi:10.2147/IJN.S198552

32. Shi X, Cheng Q, Hou T, et al. Genetically engineered cell-derived nanoparticles for targeted breast cancer immunotherapy. Mol Ther. 2020;28(2):536-547. doi:10.1016/j.ymthe.2019.11.020

33. Li J, Xu W, Yuan X, et al. Polymer-lipid hybrid anti-HER2 nanoparticles for targeted salinomycin delivery to HER2-positive breast cancer stem cells and cancer cells. Int $J$ Nanomedicine. 2017;12:6909-6921. doi:10.2147/IJN.S144184

34. Akagi T, Kaneko T, Kida T, et al. Preparation and characterization of biodegradable nanoparticles based on poly(gamma-glutamic acid) with 1-phenylalanine as a protein carrier. $J$ Control Release. 2005;108(2-3):226-236. doi:10.1016/j.jconrel.2005.08.003
35. Akagi T, Higashi M, Kaneko T, et al. Hydrolytic and enzymatic degradation of nanoparticles based on amphiphilic poly $(\gamma$-glutamic acid)-graft-1-phenylalanine copolymers. Biomacromolecules. 2006;7 (1):297-303. doi:10.1021/bm050657i

36. Lee WS, Park YH, Lee JN, et al. Comparison of HER 2 expression between primary colorectal cancer and their corresponding metastases. Cancer Med. 2014;3(3):674-680. doi:10.1002/cam4.228

37. Meric-Bernstam F, Johnson AM, Dumbrava EEI, et al. Advances in HER2-targeted therapy: novel agents and opportunities beyond breast and gastric cancer. Clin Cancer Res. 2019;25(7):2033-2041. doi:10.1158/1078-0432.CCR-18-2275

38. Nielsen UB, Kirpotin DB, Pickering EM, et al. Therapeutic efficacy of anti-ErbB2 immunoliposomes targeted by a phage antibody selected for cellular endocytosis. Biochim Biophys Acta. 2002;1591 (1):109-118. doi:10.1016/S0167-4889(02)00256-2

39. Dos Santos Giuberti C, de Oliveira Reis EC, Ribeiro Rocha TG, et al. Study of the pilot production process of long-circulating and $\mathrm{pH}$-sensitive liposomes containing cisplatin. $J$ Liposome Res. 2011;21(1):60-69. doi:10.3109/08982101003754377

40. Shukla R, Thomas TP, Peters JL, et al. HER2 specific tumor targeting with dendrimer conjugated anti-HER2 mAb. Bioconjug Chem. 2006;17(5):1109-1115. doi:10.1021/bc050348p

41. Svenson S, Tomalia DA. Dendrimers in biomedical applications reflections on the field. Adv Drug Deliv Rev. 2005;57(15):2106-2129. doi:10.1016/j.addr.2005.09.018

42. Gaucher G, Dufresne M-H, Sant VP, et al. Block copolymer micelles: preparation, characterization and application in drug delivery. J Control Release. 2005;109(1-3):169-188. doi:10.1016/j.jconrel.2005.09.034

43. Wang H, Wang Y, Xiao Z, et al. Human domain antibodies to conserved epitopes on HER2 potently inhibit growth of HER2-overexpressing human breast cancer cells in vitro. Antibodies (Basel). 2019;8(1). doi:10.3390/antib8010025.

44. Siena S, Bardelli A, Sartore-Bianchi A, et al. HER2 amplification as a 'molecular bait' for trastuzumab-emtansine (T-DM1) precision chemotherapy to overcome anti-HER2 resistance in HER2 positive metastatic colorectal cancer: the HERACLES-RESCUE trial. J Clin Oncol. 2016;34 (4_suppl):TPS774-TPS774. doi:10.1200/jco.2016.34.4_suppl.tps774

45. Oh D-Y, Bang Y-J. HER2-targeted therapies - a role beyond breast cancer. Nat Rev Clin Oncol. 2020;17(1):33-48. doi:10.1038/s41571-019-0268-3

46. Noel P, Von Hoff DD, Saluja AK, et al. Triptolide and its derivatives as cancer therapies. Trends Pharmacol Sci. 2019;40(5):327-341. doi:10.1016/j.tips.2019.03.002

47. Hou ZY, Tong X-P, Peng Y-B, et al. Broad targeting of triptolide to resistance and sensitization for cancer therapy. Biomed Pharmacother. 2018;104:771-780. doi:10.1016/j.biopha.2018.05.088
International Journal of Nanomedicine

\section{Publish your work in this journal}

The International Journal of Nanomedicine is an international, peerreviewed journal focusing on the application of nanotechnology in diagnostics, therapeutics, and drug delivery systems throughout the biomedical field. This journal is indexed on PubMed Central, MedLine, CAS, SciSearch ${ }^{\mathbb{B}}$, Current Contents ${ }^{\mathbb{B}} /$ Clinical Medicine,
Journal Citation Reports/Science Edition, EMBase, Scopus and the Elsevier Bibliographic databases. The manuscript management system is completely online and includes a very quick and fair peer-review system, which is all easy to use. Visit http://www.dovepress.com/ testimonials.php to read real quotes from published authors. 